Pacific Journal of Mathematics

FINITE DIMENSIONAL TORSION FREE RINGS 


\title{
FINITE DIMENSIONAL TORSION FREE RINGS
}

\section{Thomas J. Cheatham}

\begin{abstract}
In the class of rings with zero singular left ideal, several characterizations of rings with finite left Goldie dimension are given. They include: the direct limit of torsion free modules is torsion free; the direct limit of torsion free injective modules is injective; each absolutely pure torsion free module is injective; each module has a unique (up to isomorphism) torsion free covering module. The latter result gives a converse, in a special case, to a theorem of Mark Teply.
\end{abstract}

Throughout, $R$ will denote an associative ring with identity and module, without further qualification, will mean unitary left $R$ module. For a module $E$, we use $S \subseteq^{\prime} E$ to denote that $S$ is a large submodule of $E[4$, p. 60]; $Z(E)$ will denote the singular submodule of $E$, which consists of those elements in $E$ whose annihilators are large left ideals in $R$.

Definition 1. A module $E$ is torsion free if $Z(E)=(0)$ and if $Z(R)=(0)$ we say $R$ is a torsion free ring.

A submodule $S$ of a module $E$ is closed in $E$ if $S \subseteq^{\prime} T \subseteq E$ implies $T=S$. The following facts are easily verified.

Lemma 1. Let $S$ be a submodule of a module $E$.

(a) If $Z(E / S)=(0), S$ is closed in $E$.

(b) If $Z(E)=(0), S$ is closed in $E$ if and only if $Z(E / S)=(0)$.

Proof. See Lemma 2.3 in [8].

Definition 2. A module $E$ has finite (Goldie) dimension if it contains no infinite direct sum of nonzero submodules. If the module $R$ has finite dimension we call $R$ a finite dimensional ring and write $\operatorname{dim} R$ is finite.

1. Torsion Free Rings. Over an integral domain the direct limit of torsion free modules is torsion free. In this section we show that, in the class of torsion free rings, this property characterizes the finite dimensional rings. We also give two noetherian-like characterizations of such rings.

We record a theorem of F. Sandomierski [7] for easy reference.

THeOREM S. Let $Z(R)=(0)$, and $Q$ the maximal left quotient 
ring of $R[4, \mathrm{p} .106]$, then the following are equivalent.

(a) $Q I=Q$ for every $I \subseteq^{\prime} R$.

(b) For $I \subseteq \subseteq^{\prime} R$ there are $a_{1}, a_{2}, \cdots a_{n} \in I$ such that $\sum R a_{i} \subseteq^{\prime} R$.

(c) For $I \leqq R$ there are $a_{1}, a_{2}, \cdots a_{n} \in I$ such that $\sum R a_{i} \subseteq^{\prime} I$.

(d) $\operatorname{dim} R$ is finite.

(e) $Q$ is a semisimple (artinian) ring.

(f) $\operatorname{Ker}\left(R \otimes_{R} E \rightarrow Q \otimes_{R} E\right)=Z(E)$ for every module $E$.

Sandomierski [7, Th. 2.5, p. 118] noted that if $R$ has finite dimension the direct sum of torsion free injective modules is injective. If $Z(R)=(0)$ the converse is also known. In fact, under this assumption, it follows from Teply [10, Th. 2.1, p. 451] that $\operatorname{dim} R$ is finite if and only if any countable direct sum of torsion free injective modules is injective.

A set $\mathscr{S}$ of submodules of a module is directed if given $X, Y \in$ $\mathscr{S}$ there is a $Z \in \mathscr{S}$ such that $X \cup Y \subseteq Z$. Clearly the union of a directed set of submodules is a submodule.

We will make use of the following lemma which is an unpublished remark of M. Teply.

Lemma 2. Let $Z(R)=(0)$. The union of a directed set of closed submodules of a torsion free module is a closed submodule if and only if $\operatorname{dim} R$ is finite.

Proof. Assume that $\operatorname{dim} R$ is finite then, to show that the condition is necessary, we proceed as in [9, Prop. 2.1 (3)] using Theorem $S(b)$.

Conversely, let $E_{1}, E_{2}, \cdots$ be a countable family of torsion free injective modules. Then $E=\bigoplus_{n=1}^{\infty} E_{n}$ is a torsion free module so has a torsion free injective hull $I(E)$. But $E$ can be written as the union of the chain $S_{1} \subseteq S_{2} \subseteq \cdots$ of injective (hence closed) submodules of $I(E)$, where $S_{n}=\bigoplus_{i=1}^{n} E_{i}$. Hence $E$ is closed in $I(E)$. But $E \cong I(E)$ so $E=I(E)$, i.e., $E$ is injective. By a remark above $\operatorname{dim} R$ is finite.

The following useful result is well-known and trivial to prove.

Lemma 3. Let $f, g \in H_{0}(E, F)$ where $Z(F)=(0)$.

(1) If $f$ and $g$ agree on a large submodule of $E$ then $f=g$.

(2) If $E$ is injective, $f(E)$ is a direct summand of $F$.

Theorem 1. Let $Z(R)=(0)$. Then the following statements are equivalent.

(1) $\operatorname{dim} R$ is finite.

(2) The direct limit of torsion free modules is torsion free. 
(3) The direct limit of torsion free injective modules is injective.

Proof. (1) implies (2). Let $\left\{E, f_{a}\right\}$ be the direct limit of the directed system of torsion free modules $\left\{E_{a} ; f_{a}^{b}, A\right\}$. Then $E=\bigcup_{a \in A} \operatorname{Im}$ $f_{a}$, so to show $E$ is torsion free it suffices to show $\operatorname{Im} f_{a}$ is torsion free for each $a \in A$. But $\operatorname{Im} f_{a} \cong E_{a} / \operatorname{Ker} f_{a}$, and $\operatorname{Ker} f_{a}=\mathbf{U}_{b \geqq a} \operatorname{Ker}$ $f_{a}^{b}$. Furthermore, for each $a \in A,\left\{\operatorname{Ker} f_{a}^{b} \mid b \geqq a, b \in A\right\}$ is a directed set of submodules of the torsion free module $E_{a}$, and since $\operatorname{Im} f_{a}^{b} \subseteq$ $E_{b}$, Ker $f_{a}^{b}$ is closed in $E_{a}$. By Lemma $2 \operatorname{Ker} f_{a}$ is closed in $E_{a}$ and hence by Lemma $1(\mathrm{~b}) \operatorname{Im} f_{a}$ is torsion free. Hence (1) implies (2).

(2) implies (1). Let $\left\{C_{a}: a \in A\right\}$ be a directed set of closed submodules of a torsion free module $E$. For $a, b \in A$ such that $C_{a} \leqq C_{b}$ define a function $f_{a}^{b}: E / C_{a} \rightarrow E / C_{b}$ by $f_{a}^{b}\left(x+C_{a}\right)=x+C_{b}$. Clearly $f_{a}^{b} \in \operatorname{Hom}_{R}\left(E / C_{a}, E / C_{b}\right)$ and one easily checks that $\left\{E / C_{a} ; f_{a}^{b}, A\right\}$ is a directed system of torsion free modules with direct limit $E / \bigcup_{a \in A} C_{a}$. So from (2) $E / \cup C_{a}$ is torsion free and hence (Lemma 1(a)) $\cup_{a \in A} C_{a}$ is closed in $E$. So (1) follows from Lemma 2.

(1) implies (3). Let $\left\{E, f_{a}\right\}$ be the direct limit of a directed system of torsion free injective modules $\left\{E_{a} ; f_{a}^{b}, A\right\}$. Let $I$ be any left ideal of $R$ and $h \in \operatorname{Hom}_{R}(I, E)$. By Theorem $S(c)$ there is a finitely generated left ideal $J$ of $R$ such that $J \subseteq^{\prime} I$. From condition (2) we know that $E$ is torsion free so by Lemma $3(1)$ we see that an extension of $f^{\prime}=\left.f\right|_{J}: J \rightarrow E$ to all of $R$ will give the desired extension of $f$. To see that $f^{\prime}$ can be extended to $R$ we proceed as follows.

Let $p: F \rightarrow J$ be an $R$-homomorphism of a finitely generated free module $F$ onto $J$ and identify $J$ with $F / \operatorname{Ker} p$. It follows easily from Theorem $S$ that $F$ has finite dimension and that any submodule of $F$ has a finitely generated large submodule. Thus let $K$ be a finitely generated large submodule of Ker $p$.

Now $f^{\prime} \circ p(F)$ is a finitely generated submodule of $E=\mathbf{U}_{a \in A} \mathrm{Im}$ $f_{a}$ so there's an $a \in A$ such that $f^{\prime} \circ p(F) \subseteq \operatorname{Im} f_{a}$. Hence, by the projectivity of $F$, there's an $h \in \operatorname{Hom}_{R}\left(F, E_{a}\right)$ such that $f_{a} \circ h=f^{\prime} \circ p$. Then $h\left(\operatorname{Ker} f^{\prime} \circ p\right) \leqq \operatorname{Ker} f_{a}=\bigcup_{b \geqq a} \operatorname{Ker} f_{a}^{b}$ and since $K$ is finitely generated $h(K) \subseteq \operatorname{Ker} f_{a}^{b}$ for some $b \geqq a$, i.e., $f_{a}^{b} \circ h(K)=(0)$. Therefore $f_{a}^{b} \circ h$ induces an $R$-homomorphism $g$ from $F / \operatorname{Ker} p=J$ into $E_{b}$. But $E_{b}$ is injective so $g$ can be extended to $g^{*} \in \operatorname{Hom}_{R}\left(R, E_{b}\right)$. Define $f^{*}$ from $R$ to $E$ by $f^{*}=f_{b} \circ g^{*}$. Then $f^{*}$ is an extension of $f^{\prime}$ so the desired extension of $f$.

(3) implies (1). Clearly (3) implies that any direct sum of torsion free injective modules is injective. So (1) follows by a previous remark. This completes the proof. 
As a corollary we give an easy proof of (d) implies (e) in Theorem S.

Corollary. A finite dimensional torsion free ring $R$ has a semisimple (artinian) maximal left quotient ring $Q$.

Proof. It suffices to show that every left ideal of $Q$ is a direct summand of $Q$ (as a $Q$-module). Since $Q$ is von Neumann regular any finitely generated left ideal of $Q$ is a direct summand. Hence any such ideal is left $R$-injective as $Q$ is. But $Z\left({ }_{R} Q\right)=(0)$ and it follows, via a direct limit argument and Theorem 1(3), that any left ideal of $Q$ is $R$-injective, hence $Q$-injective. Thus any left ideal of $Q$ is a direct summand.

B. Maddox [5] calls a module $M$ absolutely pure if for every module $E$ containing $M$ as a submodule the sequence $0 \rightarrow G \otimes M \rightarrow$ $G \otimes E$ is exact for every right $R$-module $G$. He showed that any direct sum of absolutely pure modules is absolutely pure. C. Megibben [6, Th. 3, p, 564] characterized left noetherian rings by the property "each absolutely pure module is injective." This result was also obtained indepently by Edgar Enochs.

We have a corresponding characterization of finite dimensionality in the class of torsion free rings.

Theorem 2. Let $Z(R)=(0)$. Then $\operatorname{dim} R$ is finite if and only if each absolutely pure torsion free module is injective.

Proof. Assume that $\operatorname{dim} R$ is finite, and let $E$ be an absolutely pure torsion free module and $f$ an $R$-homomorphism of a left ideal $I$ of $R$ into $E$. Let $J$ be a finitely generated left ideal of $R$ such that $J \subseteq \subseteq^{\prime} I$. As remarked above it suffices to extend $f^{\prime}$, the restriction of $f$ to $J$, to all of $R$. But this can be done by [6, Cor. 2, p. 562].

Conversely, it suffices to show that the direct sum of torsion free injective modules is injective. But any such sum is torsion free and absolutely pure, hence injective.

2. Torsion free covers. The main result of this section (Theorem 3) gives a converse to $[9$, Th. 2.4, p. 459], in a special case. We begin with a definition.

Definition 3. A torsion free cover of a module $E$ is a homomorphism $g$ from a torsion free module $T(E)$ onto $E$ such that:

(1) Ker $g$ contains no nonzero closed submodule of $T(E)$, 
(2) given $f \in \operatorname{Hom}_{R}(F, E)$ where $F$ is torsion free there is an $h \in \operatorname{Hom}_{R}(E, T(E))$ such that $g \circ h=f$.

This definition was given initially for modules over an integral domain by E. Enochs [3] who proved that, in this case, every module has a unique (up to isomorphism) torsion free covering module. B. Banaschewski [1, p. 59] gave the following construction for the cover of a module $E$ over an integral domain $R$ with quotient field $K$ : $T(E)=\left\{f \in \operatorname{Hom}_{R}(K, I(E)) \mid f(1) \in E\right\} ; g(f)=f(1)$.

M. Teply [8, p. 449] generalized the notion of a torsion free cover to a hereditary torsion theory $(\mathscr{T}, \mathscr{F}$ ) [2] of $R$-modules. He proved that each module has a $\mathscr{T}$-torsion free cover if $R \in \mathscr{F}$ and the direct sum of $\mathscr{T}$-torsion free injective modules is injective.

The Goldie torsion theory $(\mathscr{G}, \mathscr{F})$ is the torsion theory whose torsion class $\mathscr{G}$ is generated by all factor modules $B / A$ where $A$ is a large submodule of $B$. The Goldie torsion free class $\mathscr{F}$ is precisely the class of torsion free modules given by Definition 1 . Teply's result shows that $\mathscr{G}$-torsion free covers exist if $Z(R)=(0)$ and $\operatorname{dim}$ $R$ is finite. We prove the converse and show that Banaschewski's construction has an obvious analogy in this case.

THEOREM 3. Let $R$ be a ring with identity and maximal left quotient ring $Q$. If every left $R$-module has a torsion free cover then $Z(R)=(0)$ and $\operatorname{dim} R$ is finite. Moreover, the evaluation map from $T(E)=\left\{f \in \operatorname{Hom}_{R}(Q, I(E)) \mid f(1) \in E\right\}$ onto $E$ is a torsion free cover of $E$.

Proof. Let $(0) \rightarrow \operatorname{Ker} g \rightarrow T(R) \stackrel{g}{\longrightarrow} R \rightarrow(0)$ be a torsion free cover of the module $R$. Since $R$ is projective this sequence splits and hence Ker $g$ is closed in $T(R)$. Then Ker $g=(0)$ as $g$ is a cover of $R$ so $R \cong T(R) \in \mathscr{F}$, i.e., $Z(R)=(0)$.

To show that $\operatorname{dim} R$ is finite it suffices, by Theorem $\mathrm{S}$, to show that $Q$ is a semisimple ring. But, since $Z(R)=(0), Q$ is von Neumann regular so it suffices to show that $Q$ is a finite dimensional ring. If not, there is an infinite set of nonzero elements $\left\{x_{i}: i \in I\right\}$ of $Q$ such that the sum $B=\sum Q x_{i}$ is direct and a proper large left ideal of $Q$. Then $Q / B \neq(0)$ has a torsion free cover (as an $R$-module), say $g: F \rightarrow Q / B$.

Since $Z\left({ }_{R} Q\right)=(0)$ the natural $R$-homomorphism $p: Q \rightarrow Q / B$ factors thru $g$, i.e., there exist $h \in \operatorname{Hom}_{R}(Q, F)$ such that $g \circ h=p$. Then $h(B) \subseteq$ Ker $g$ and $p \neq 0$ implies $h \neq 0$ so by Lemma $3(1) h(B) \neq(0)-$ we must note that $B$ is a large $R$-submodule of $Q$. Then $h\left(Q x_{j}\right) \neq$ (0) for some index $j \in I$. By Lemma $3(2) h\left(Q x_{j}\right)$ is a direct summand of $F$. Thus we have a nonzero closed submodule of $F$ contained in 
Ker $g$. This gives a contradiction so $Q$ is a finite dimensional ring, hence semisimple.

Since $Q$ is a semisimple ring it follows that any $Q$-module is torsion free when considered as an $R$-module. Hence any $R$-submodule of such a module is a torsion free $R$-module. Conversely, it follows from Theorem $S(f)$ that any torsion free $R$-module is an $R$-submodule of a $Q$-module (i.e., is $Q$-extendible in Banaschewski's terminology). Banaschewski [1, p. 63] established the existence of $Q$-extendible coverings.

Note that if $q_{1}, q_{2}, \cdots, q_{n} \in Q$, and $f_{1}, f_{2}, \cdots, f_{n} \in T(E)=\left\{f \in \operatorname{Hom}_{R}(Q\right.$, $I(E)) \mid f(1) \in E\}$ are such that $\sum q_{i} f_{i}=0 \in \operatorname{Hom}_{R}(Q, I(E))$ then the large left ideal $I=\left\{r \in R \mid r \quad q_{i} \in R\right.$ for all $\left.i\right\}$ annihilates $\sum q_{i} \otimes f_{i}$ in $Q \otimes_{R} T(E)$. Thus $\sum q_{i} \otimes f_{i} \in Z\left(Q \otimes_{R} T(E)\right)=(0)$. This shows that the $R$-homomorphism $Q \bigotimes_{R} T(E) \rightarrow \operatorname{Hom}_{R}(Q, I(E))$ given by the $Q$ module structure of $\operatorname{Hom}_{R}(Q, I(E))$ is one-to-one. Therefore by [1, Prop. 3, p. 64] the evaluation map from $T(E)$ onto $E$ is a torsion free cover of $E$. This completes the proof.

REMARK. This theorem shows that "torsion free" covers do not exist for the ring mentioned by Banaschewski [1, p. 66], that is, the ring of all functions on a set $X$ with values in a field which are constant except on some finite set. Since $Z(R)=(0)$ the "torsion free" he is using agrees with our torsion free and covers do not exist.

This paper constitutes a portion of the author's doctoral dissertation at the University of Kentucky. The author is deeply indebted to Professor Edgar Enochs, his advisor, for his generous advice and encouragement.

\section{REFERENCES}

1. B. Banaschewski, On covering of modules, Math. Nachr., 31 (1966), 57-71.

2. S. E. Dickson, A torsion theory for abelian categories, Trans. Amer. Math. Soc., 121 (1966), 223-225.

3. E. Enochs, Torsion free covering modules, Proc. Amer. Math. Soc., 14 (1963), 884-889.

4. J. Lambek, Lectures on rings and modules, Blaisdell Publishing Co., Waltham, Mass., 1966.

5. B. Maddox. Absolutely pure modules, Proc. Amer. Math. Soc., 18 (1967), 155-158.

6. C. Megibben, Absolutely pure modules, Proc. Amer. Math. Soc., 26 (1970), 561-566.

7. F. Sandomierski, Semisimple maximal quotient rings, Trans. Amer. Math. Soc., 128 (1967), 112-120.

8. F. Sandomierski, Nonsingular rings, Proc. Amer. Math. Soc., 19 (1968), 225-230.

9. M. Teply, Torsion free injective modules, Pacific J, Math., 28 (1969), 441-453.

10. - Some aspects of Goldie's torsion theory, Pacific J. Math., 29 (1969), 447-459.

Received April 2, 1971 and in revised form July 15, 1971. 


\section{PACIFIC JOURNAL OF MATHEMATICS}

\section{EDITORS}

H. SAMELSON

Stanford University

Stanford, California 94305

C. R. HobBY

University of Washington

Seattle, Washington 98105
J. DugundjI

Department of Mathematics

University of Southern California

Los Angeles, California 90007

RICHARD ARENS

University of California

Los Angeles, California 90024

\section{ASSOCIATE EDITORS}

E. F. BECKENBACH

B. H. NeUMaNN

F. WOLF

K. YoshidA

\section{SUPPORTING INSTITUTIONS}

UNIVERSITY OF BRITISH COLUMBIA

CALIFORNIA INSTITUTE OF TECHNOLOGY

UNIVERSITY OF CALIFORNIA

MONTANA STATE UNIVERSITY

UNIVERSITY OF NEVADA

NEW MEXICO STATE UNIVERSITY

OREGON STATE UNIVERSITY

UNIVERSITY OF OREGON

OSAKA UNIVERSITY
UNIVERSITY OF SOUTHERN CALIFORNIA STANFORD UNIVERSITY

UNIVERSITY OF TOKYO

UNIVERSITY OF UTAH

WASHINGTON STATE UNIVERSITY UNIVERSITY OF WASHINGTON

$\stackrel{*}{*} \stackrel{*}{*} \stackrel{*}{*}$ AMERICAN MATHEMATICAL SOCIETY
NAVAL WEAPONS CENTER 


\section{Pacific Journal of Mathematics}

Vol. 39 , No. 1

May, 1971

Charles A. Akemann, A Gelfand representation theory for $C^{*}$-algebras ....

Sorrell Berman, Spectral theory for a first-order symmetric system of

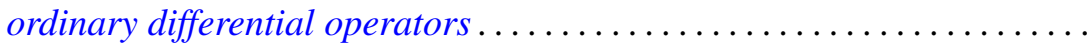

Robert L. Bernhardt, III, On splitting in hereditary torsion theories ........

J. L. Brenner, Geršgorin theorems, regularity theorems, and bounds for determinants of partitioned matrices. II. Some determinantal identities ..........................................

Robert Morgan Brooks, On representing $F^{*}$-algebras .............. 51

Lawrence Gerald Brown, Extensions of topological groups........... 71

Arnold Barry Calica, Reversible homeomorphisms of the real line ........ 79

J. T. Chambers and Shinnosuke Oharu, Semi-groups of local Lipschitzians in

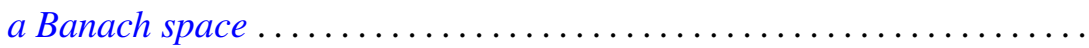

Thomas J. Cheatham, Finite dimensional torsion free rings .............

Byron C. Drachman and David Paul Kraines, A duality between

transpotence elements and Massey products ...................

Richard D. Duncan, Integral representation of excessive functions of a

Markov process ......................................

George A. Elliott, An extension of some results of Takesaki in the reduction

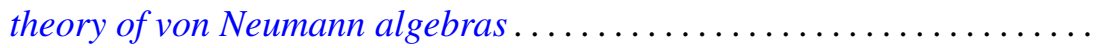

Peter C. Fishburn and Joel Spencer, Directed graphs as unions of partial

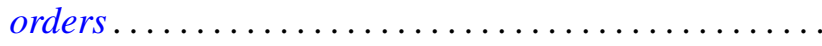

Howard Edwin Gorman, Zero divisors in differential rings ...

Maurice Heins, A note on the Löwner differential equations...

Louis Melvin Herman, Semi-orthogonality in Rickart rings. .

David Jacobson and Kenneth S. Williams, On the solution of linear G.C.D.

equations

Michael Joseph Kallaher, On rank 3 projective planes ... . .

Donald Paul Minassian, On solvable $O^{*}$-groups ...........

Nils Øvrelid, Generators of the maximal ideals of $A(\bar{D})$

Mohan S. Putcha and Julian Weissglass, A semilattice decomposition into

semigroups having at most one idempotent ............

Robert Raphael, Rings of quotients and $\pi$-regularity ....

J. A. Siddiqi, Infinite matrices summing every almost periodic sequence. .

Raymond Earl Smithson, Uniform convergence for multifunctions ...

Thomas Paul Whaley, Mulitplicity type and congruence relations in

universal algebras...

Roger Allen Wiegand, Globalization theorems for locally finitely generated modules... 